\title{
HATE SPEECH DETECTION IN TWEETS USING MACHINE LEARNING ALGORITHM
}

\author{
Mrs. KopparthiHarika \\ Pragati Engineering College, \\ Kakinada, Andhra Pradesh, India \\ CH Mounika, T Anuradha, P Sharon, \\ B.Tech, Computer Science and Engineering, \\ Pragati Engineering College, \\ Kakinada, Andhra Pradesh, India,
}

\begin{abstract}
Hate speech is currently of broad and current interest in the domain of social media. The anonymity and flexibility afforded by the Internet has made it easy for users to communicate in an aggressive manner. And as the amount of online hate speech is increasing, methods that automatically detect hate speech is very much required. Moreover, these problems have also been attracting the Natural Language Processing and Machine Learning communities a lot. Therefore, the goal of this paper is to look at how Natural Language Processing applies in detecting hate speech. Furthermore, this paper also applies a current technique in this field on a dataset.
\end{abstract}

\section{INTRODUCTION}

Natural Language Processing (NLP) of NLP is sentiment analysis. From opinion polls to creating entire marketing strategies, this domain has completely reshaped the way businesses work, which is why this is an area every data scientist must be familiar with. In order to create a hatespeech-detecting algorithm, we are going to use Python-based NLP machine learning techniques. Machine learning is basically teaching machines to accomplish various tasks by training them through data. In this case, we are going to collect data from the Korean radical anti-male website, Womad, but you are free to use different kinds of data as long as the data is labeled appropriately (more on that later). Then, using a NLP (or Natural Language Processing) technique called Tf-Idf vectorization, well extract keywords that convey importance within hate speech.

\section{PROPOSED AlgORITHM}

Methodology of sentimental analysis in twitter mainly involves 5 steps.

\subsection{DATA COLLECTION/ TWEET EXTRACTION}

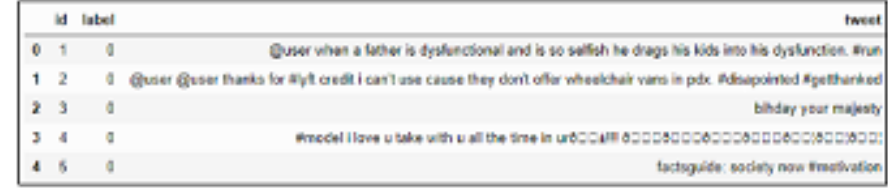

Fig. Tweets Preprocessing and cleaning

Twitter API - A Python wrapper for performing API requests. For fetching the twitter data from the twitter API includes the following steps 1] Installation of the needed software 2] authentication of twitters data. The main installation softwares include tweepy, text blob, nltk etc, Authentication involves different stepsstep1: visit the twitter website and click the button ' create new app' . Step2:fill the details in the form provided and submit.Step3:It will be redirected to the app page where the " ' consumer keys' , ' consumer access', ' access token' and ' access token secret' " that is needed to access the twitter data will be present.Step4:implement in python. There are different sources for storing the data taken from the twitter. They are like MongoDB, open source document storage database and is the go-to "No SQL" database. It makes working with a database feel like working with JavaScript. PyMongo, a Python wrapper for interfacing with a MongoDB instance. This library lets you connect your Python scripts with your database and read/insert records. This is an example of the data that is been extracted from the twitter on the topic

\subsection{PRE-PROCESSING}

Once the data is collected from the twitter the next step is preprocessing that is implemented in python. There are several steps involved in the preprocessing stage. They are,

1. Converting all uppercase letters to lowercase.

2. Tokenization generally done by installing the NLP package. It generally means removal of hash tags, numbers (1, 2, 3 etc.,), URL's and targets (@). Once 


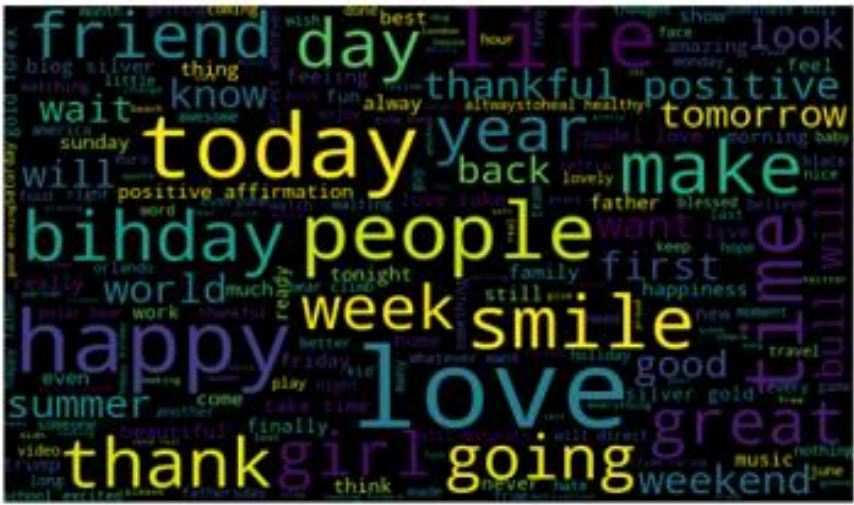

Fig. Understanding the common words used in the tweets

We can see most of the words are positive or neutral. With happy and love being the most frequent ones. It doesn't give us any idea about the words associated with the racist/sexist tweets. Hence, we will plot separate wordclouds for both the classes (racist/sexist or not) in our train data.

\section{B. Words in non racist/sexist tweets}

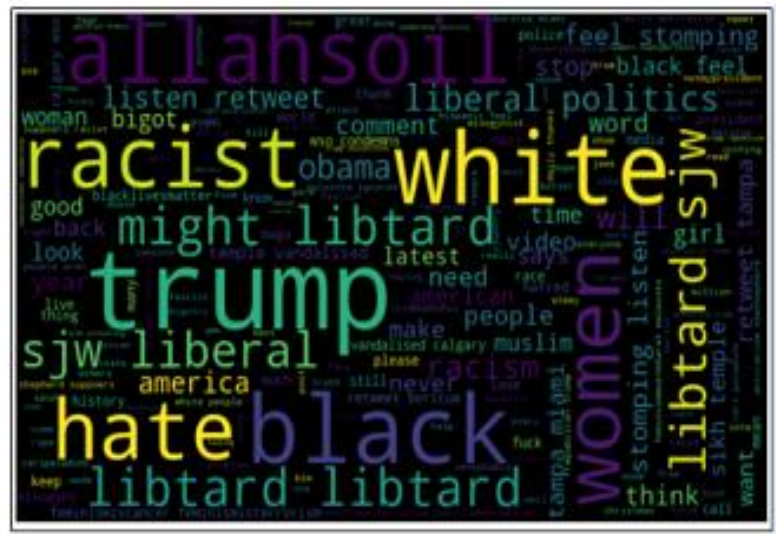

Fig 4.2.5 reciutsexeist meets

We can see most of the words are positive or neutral. With happy, smile, and love being the most frequent ones. Hence, most of the frequent words are compatible with the sentiment which is non racist/sexists tweets. Similarly, we will plot the word cloud for the other sentiment. Expect to see negative, racist, and sexist terms.

\section{Racist/Sexist Tweets}

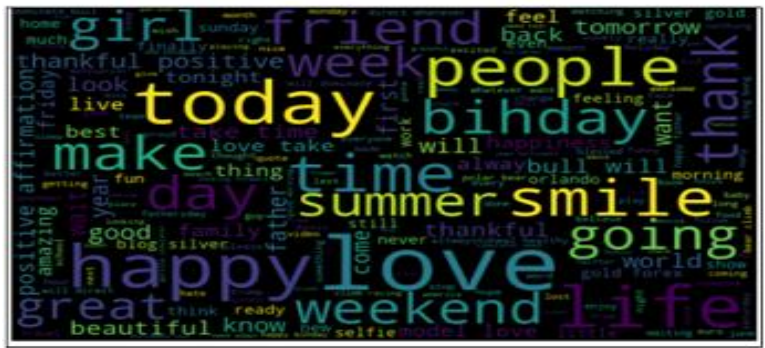

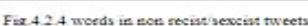

As we can clearly see, most of the words have negative connotations. So, it seems we have a pretty good text data to work on. Next we will the hashtags/trends in our twitter data.

\section{Non-Racist/Sexist Tweets}

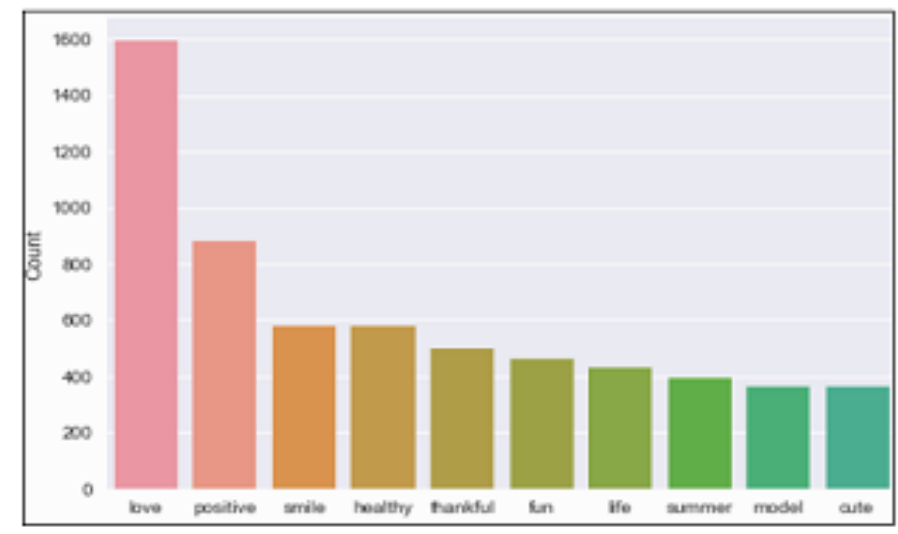

Fig 4.2 .6 hashtags appearing in the noe racist sexist tweets.

All these hashtags are positive and it makes sense. I am expecting negative terms in the plot of the second list. Let's check the most frequent hashtags appearing in the racist/sexist tweets.

\section{E. Racist/Sexist Tweets}

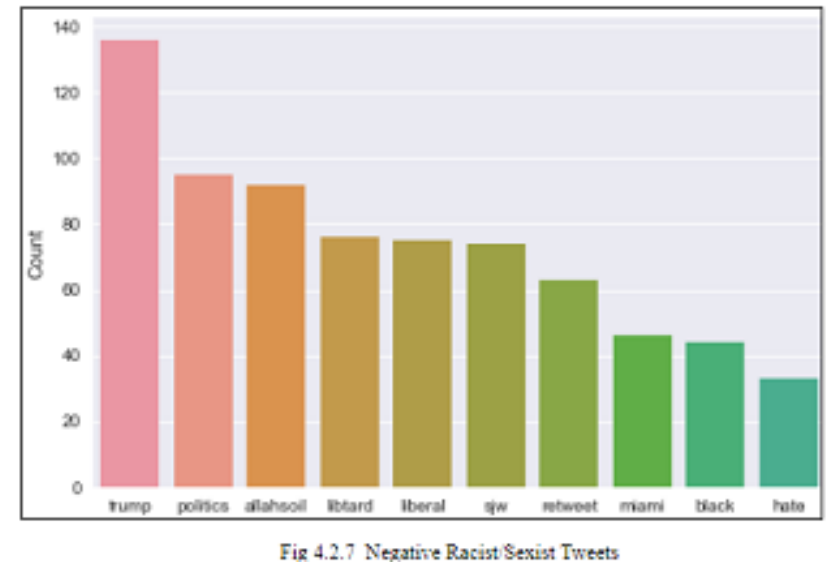

As expected, most of the terms are negative with a few neutral terms as well. So, it's not a bad idea to keep these hashtags in our data as they contain useful information. Next, we will try to extract features from the tokenized tweets.

\section{CONCLUSION}

In this paper, the aim was to detect hate speech using a Natural Language Processing technique. To enable successful execution of the research it was first necessary to understand what hate speech is. To accomplish this, an overview of this topic has been conducted. Here it can be 


\section{International Journal of Engineering Applied Sciences and Technology, 2020 \\ Vol. 4, Issue 12, ISSN No. 2455-2143, Pages 558-561 \\ Published Online April 2020 in IJEAST (http://www.ijeast.com)}

concluded that hate speech has several definitions, all coming from different platforms. Hate speech detection is a classification-related task, and that's why further literature was reviewed to understand the idea behind Natural Language Processing and the application of various techniques. Previous work showed that deep learning models improve the state-of-art approaches within hate speech classification tasks. Therefore, a deep learning method, namely a Convolutional Neural Network (CNN), has been applied on a Twitter dataset. This data contains tweets annotated with three labels: hate, offensive language and neither.

\section{REFERENCE}

1) M. Ott, Y. Choi, "Finding deceptive opinion spam by any stretch of the imagination; Proceedings of the Annual Meeting of the Association for Computational Linguistics: Human Language Technologies-Volume; pp. 309-319, 2011;

2) C. Cardie, J.T. Hancock Negative Deceptive Opinion Spam; Proceedings of the HLT-NAACL; pp. 497-501, 2013.

3) C.H. Yu , M.W. Ward, M. Morabito, W. Ding, Crime forecasting using data mining techniques; Proceedings of the International Conference on Data Mining, ICDM'11; Vancouver, BC, CanadaCanada, pp. 779-789, 2011;

4) A. Bermingham, A. Smeaton, On using Twitter to monitor political sentiment and predict election results; Proceedings of the Workshop on Sentiment Analysis Where AI meets Psychology (SAAIP 2011); Chiang Mai, Thailand, pp. 2-10, 2011;

5) X. Wang , D. W. Brown , M. S. Gerber, Spatiotemporal modeling of criminal incidents using geographic, demographic, and Twitter-derived information; Proceedings of the 2012 IEEE International Conference on Intelligence and Security Informatics (ISI); Arlington, pp. 36-41, 2012;

6) J. Bollen , H. Mao, X. Zeng, Twitter mood predicts the stock market. J. Comput, pp. 1-8, 2011;

7) P.T. Metaxas , E Mustafaraj , D. Gayo-AvelloGayoAvello, How (not) to predict elections; Proceedings of the 2011 IEEE Third International Conference on Privacy, Security, Risk and Trust and 2011 IEEE Third International Conference on Social Computing; Boston, pp. 165-171, 2011;

8) M. Ott, C. Cardie , J. K. Hancock, Negative Deceptive Opinion Spam; Proceedings of the HLTNAACL, pp. 497-501, 2013;

9) A.K. Uysal , S. Gunal, A novel probabilistic feature selection method for text classification. Knowl.Based Syst, pp. 226-235, 2012;
10) D.M.W. Powers, Evaluation: From precision, recall and f-measure to roc., informedness, markedness \& correlation, pp. 37-63, 2011;

11) M.L. McHugh, Interrater reliability: The kappa statistic. Biochem. Med. Biochem. Med. 22:276-282. doi: 10.11613/BM.2012.031, 2012;

12) J. Heidemann, M. Klier, F. Probst, Online social networks: A survey of a global phenomenon.doi: 10.1016/j.comnet.2012

13) D. Choudhury, A. Paul, Community detection in social networks: An Over, pp. 6-13, 2013;

\section{ACKNOWLEDGMENT}

It gives us an immense pleasure to express deep sense of gratitude to our guide Mrs. Kopparthi Harika, Department of Computer Science \& Engineering because of his whole hearted and invaluable guidance throughout the report. Without his sustained and sincere effort, this report would not have taken this shape. She encouraged and helped us to overcome various difficulties that we have faced at various stages of our report.

We would like to sincerely thank Dr. M. Radhika Mani, Professor \& HOD,

Computer Science \& Engineering, for providing all the necessary facilities that led to the successful completion of our report.

We would like to take this opportunity to thank our beloved Principal and Vice

Principal Dr. S. Sambhu Prasad, Dr. K. Satyanarayana for providing a great

support to us in completing our project and for giving us the opportunity of doing the mini project report. 